\title{
Effects of Tibialis Anterior Muscle Vibration on Quiet Stance
}

\author{
David R. Temple*, Beom-Chan Lee, $\mathrm{PhD}^{\dagger}$, and Charles S. Layne, $\mathrm{PhD}^{\ddagger}$ \\ Department of Health and Human Performance \\ Center for Neuromotor and Biomechanics Research
}

University of Houston

\begin{abstract}
Understanding the context in which vibration of musculature about the ankle joints elicits postural sway is important if it is to be utilized as a means of manipulating postural control for therapeutic or training purposes. The purpose of this study was to observe if the postural response associated with the vibration of the tibialis anterior (TA) muscles would remain intact despite multiple exposures during a series of forward and backward translating perturbations. Twenty young healthy adults (18-35 years old) were asked to maintain an upright erect posture on a forceplate with their eyes closed during two separate trials of quiet stance both without (preNV) and with (preVib) TA muscle vibration being applied. Subjects were then exposed to 60 bouts of forward and backwards translating perturbations of various amplitudes with TA vibration being applied during 30 of the perturbations, after which another trial of quiet stance with TA vibration (postVib) was taken. Anterior-posterior center of pressure (COP) and shear force measurements were used to calculate root-mean-square (RMS) of the sway, mean power frequency (MPF), shift from zero, and strategy score percentages as dependent variables. Of interest here are the postural responses obtained during the quiet stance conditions. A one-way ANOVA with Tukey's HSD post-hoc tests were used to compare means of the three quiet stance conditions for each of the dependent variables. Non-significant MPF results revealed no change in sway frequency oscillations due to vibration throughout the study. RMS sway was significantly greater for preVib and postVib trials than preNV, and strategy scores were significantly lower for preVib and postVib than preNV, indicating greater shear forces with the vibration conditions. No significant differences between preVib and postVib means were observed in any of the COP measures. This indicates that adaptation to the TA muscle vibration response did not occur and suggests that the distorted input associated with vibration was not fully down weighted by the sensory motor system during quiet upright stance, despite repeated exposure to vibration throughout the testing protocol.
\end{abstract}

\footnotetext{
* e-mail: drtemple@uh.edu

† e-mail: blee24@central.uh.edu

* e-mail: clayne2@central.uh.edu
}

IEEE Haptics Symposium 2014 23-26 February, Houston, Tx, USA

978-1-4799-3131-6/14/\$31.00 @2014 IEEE
Keywords: muscle vibration, postural control, postural response, postural sway, proprioception, somatosensation.

\section{INTRODUCTION}

To remain upright during quiet stance, the projection of the center of gravity (COG) must be kept within the area bounded by the base of support $[1,2]$. Maintaining balance requires the central nervous system (CNS) to utilize an interaction of sensory information from the visual, vestibular, and somatosensory systems to make appropriate postural adjustments via closed-loop processes [1, 3-8]. It has been suggested that the CNS has the ability to ignore inaccurate or irrelevant stimuli from certain sensory systems in favor of relying on more accurate information from other sensory systems via a process known as sensory reweighting $[3,9]$.

Muscle vibration has frequently been used to manipulate the proprioceptive input provided by the muscles being vibrated. It has been shown that mechanical vibration preferentially stimulates the primary afferent fibers (Type Ia) of muscle spindles when applied to muscle tendons or directly to the muscle [3, 10-16]. This stimulation often causes a tonic vibration reflex (TVR) [10-16] as well as generates proprioceptive misinformation on the vibrated muscles' lengths, and therefore creates a perception the muscles are longer than they actually are $[3,10]$. Evoking this inaccurate proprioceptive input about musculature controlling the ankle joints often results in anterior-posterior $(\mathrm{A} / \mathrm{P})$ postural sways being generated during quiet stance, which can be observed with center of pressure (COP) measurements obtained with a force plate $[2,5,17$ 19]. For example, Achilles tendon vibration typically evokes posterior COP trajectories, while tibialis anterior (TA) muscle vibration tends to elicit more anterior sway [17-19].

If postural responses can be controlled by vibrating specific musculature, then utilizing vibration as a means of manipulating posture control may have several practical applications for use in therapeutic and training programs designed to improve balance and mobility. However, some evidence suggests that adaptation can occur to the postural responses elicited by vibration such that sustained vibration no longer generates the postural sway previously observed [3, 17]. This adaptation could potentially occur as a result of sensory down weighting of unreliable somatosensory afferents, but it appears to be context 
specific with the tasks and goals of an individual largely affecting their response to vibration $[3,17,20$, 21]. The purpose of this study was to determine if the typical pattern of forward sway generated by TA vibration during quiet stance remained intact despite extended exposures to TA vibration during a series of forward and backward translations. We hypothesized that the vibration response during quiet upright stance as assessed by several COP measures would remain relatively unchanged despite repeated exposure to vibration during the perturbation trials. This hypothesis was based on the premise that the amount of sway induced by TA vibration was not threatening to an individual's overall stability, and therefore the context in which inaccurate sensory input was present did not require a down weighting (i.e. effective ignoring) of the inaccurate sensory input.

\section{METHOD}

\subsection{Subjects}

Twenty young, healthy adults $(15$ males, 5 females; mean age $25.8 \pm 3.9$ yrs.) participated in this study. Subjects were excluded if they had any known neurological dysfunction, heart conditions, blood pressure irregularities, breathing difficulties, bone or joint issues, were pregnant, had diabetes, were epileptic, had balance problems or had any major operations recently that may affect their balance as reported through questioning and by a physical activity readiness questionnaire (PAR-Q). Subjects were also excluded if they were over the age of 35 . Prior to data collection, all subjects provided informed consent, and approval to conduct this study was granted by the Committees for the Protection of Human Subjects (CPHS) at the University of Houston.

\subsection{Instrumentation}

The projected COP displacements were measured by a computer-controlled, hydraulically driven force plate platform system (Equitest; NeuroCom International, Clackamas, OR). COP displacement data was sampled at a rate of $100 \mathrm{~Hz}$. In addition to recording COP displacement during static trials, the NeuroCom system also produced low, medium, and high translating perturbations in the forwards and backwards directions at amplitudes of 2.032, 4.064, and 6.35 centimeters respectively with a constant velocity of 15.875 centimeters per second, regardless of amplitude or direction.

Two portable vibrators (VB115; Techno-Concept, Cereste, France) were used to provide the sensory stimulus to the TA muscles. The vibrators were attached bilaterally to the belly of the muscles with rubber bands. Frequency of the vibrators was set at $80 \mathrm{~Hz}$ for all conditions involving TA vibration.

\subsection{Procedure}

Prior to testing, measures of height and weight were obtained using a common physician's mechanical column scale.
During testing, subjects stood barefoot on the NeuroCom platform with their eyes closed, arms held across their chest, vibrators attached, and feet spaced apart at a width specified by the NeuroCom's instruction manual [22], which was determined by height. Subjects were verbally reminded to keep their eyes closed, arms across their chest, and stand up as straight as possible before every trial. Trials did not begin until subjects signaled they were ready and the COP trace was stable. The first trial consisted of 12 seconds of static stance without any vibration or perturbations occurring (preNV). The second trial also consisted of 12 seconds of static stance; however, the vibrators were activated four seconds into the trial, allowing for eight seconds of data to be collected with TA vibration (preVib). The subjects were then exposed to 12 blocks of five translating perturbation trials with varying perturbation amplitudes (high, medium, or low), direction (forwards or backwards), and TA vibration either present or absent. To perform all 60 perturbation trials required approximately 35 minutes, with vibration being applied for half of the trials. Once all perturbation trials were completed, a final 12 second static balance post-test trial with TA vibration being applied four seconds into the trial (postVib) was performed to determine if the subjects remained responsive to vibration as assessed by $\mathrm{A} / \mathrm{P}$ $\mathrm{COP}$ and shear force measures. Each subject was asked to rest their body for approximately $20 \mathrm{~s}$ between trials in each static condition.

\subsection{Data Analysis}

Only data for the static upright stance trials is contained in this report as the current focus is to determine if repeated exposure to TA vibration modifies the postural sway during quiet stance. Data for all the static trials (preNV, preVib, and postVib) was processed using MATLAB (The Math Works, Natick, MA). A/P COP trajectories were filtered using a $10 \mathrm{~Hz}$ low pass filter, and demeaned to a point zero based upon the mean of the first second of data collected. The data from the final eight seconds of the trials was then processed and analyzed.

Dependent variables included root-mean-square (RMS) of the sway, mean power frequency (MPF), and shift from zero of the A/P COP trajectories. Shift from zero was calculated by taking the average position of the A/P COP trajectories to observe how far the subjects may have shifted from the zero point in response to the vibration conditions. Positive shift from zero positions indicate anterior sway, while negative shift from zero positions would represent posterior sway. A/P shear force data was used to calculate a strategy score percentage based on the equation from the NeuroCom clinical interpretation guide [23]. Equation 1 shows the calculation for strategy score.

$$
\text { Strategy Score }=\left[1-\frac{S H_{\max }-S H_{\min }}{11.4}\right] \times 100
$$

SHmax represents the maximum shear force limit while SHmin is the minimum shear force limit, and 
thus the peak-to-peak amplitude of shear oscillation is utilized in the equation. The value of 11.4 indicates the maximum possible amplitude of shear force oscillation being 11.4 kilograms. Higher strategy scores depict less shear force and are associated with ankle strategies (i.e. inverted pendulum-like sway around the ankles), while lower strategy scores are associated with greater shear forces and result in hip strategies (i.e. increased motion about the hips relative to ankle strategies).

A one-way ANOVA was utilized to compare the main effects of static condition (preNV, preVib, and postVib) on each dependent variable (RMS sway, strategy score, shear force range amplitude, MPF, and shift from zero) using IBM SPSS Statistics 20.0. Hypotheses for the main effects of condition were tested using an F-test. To determine which factors influenced the main effects, post-hoc tests (Tukey's HSD) were conducted. The level of significance was set at $p<0.05$.

\section{RESULTS}

Means, standard deviations, and standard errors of the three static conditions (preNV, preVib, and postVib) for all dependent variables are reported in Table 1 . Table 2 shows the main effects of the static condition for each of the dependent variables. Pairwise comparisons of the three static conditions are also reported in Table 2. Figure 1 shows the group mean and variability in the A/P COP trajectories across all subjects as a function of the preNV and preVib conditions. More variability of the A/P COP trajectories was observed in the preVib condition than that of the preNV condition.

Figure 2 displays the means and standard errors of RMS sway for the three static conditions. The oneway ANOVA yielded significant differences between group means for RMS Sway $(F(2,57)=10.43, p<$ $0.001)$. Post hoc analysis revealed significantly greater RMS sway means for the preVib $(p=0.001)$ and postVib $(p<0.001)$ static conditions when compared to the preNV condition means; however, there were no significant differences $(p=0.911)$ for RMS sway between the preVib and postVib conditions.

Figure 3 displays the means and standard errors of strategy score percentage for the three static conditions. Statistically significant group mean differences were also found for strategy score $(F(2,57)=4.67, p=0.013)$ with the one-way ANOVA. Post hoc analysis showed significantly greater strategy scores for the preNV condition when compared to the preVib $(p=0.018)$ and postVib $(p=$ 0.048 ) conditions. However, there were no significant differences $(p=0.915)$ for the strategy scores between the preVib and postVib conditions.

There were no statistically significant differences observed between group means of the three static conditions for MPF $(F(2,57)=0.35, p=0.71)$ or shift from zero $(F(2,57)=0.54, p=0.58)$.

\begin{tabular}{cccc}
\hline \hline \multirow{2}{*}{ Dependent Variable } & $\begin{array}{c}\text { Static } \\
\text { Condition }\end{array}$ & Mean & St. Error \\
\hline \multirow{3}{*}{ RMS Sway (cm) } & PreNV & 0.737 & 0.095 \\
& PreVib & 1.741 & 0.155 \\
& PostVib & 1.852 & 0.275 \\
\hline \multirow{2}{*}{ Strategy Score (\%) } & PreNV & 95.917 & 0.473 \\
& PreVib & 93.958 & 0.528 \\
& PostVib & 94.237 & 0.469 \\
\hline \multirow{2}{*}{ MPF (Hz) } & PreNV & 0.294 & 0.041 \\
& PreVib & 0.281 & 0.033 \\
& PostVib & 0.322 & 0.032 \\
\hline \multirow{2}{*}{ Shift From Zero (cm) } & PreNV & 0.231 & 0.157 \\
& PreVib & 0.424 & 0.304 \\
& PostVib & 0.658 & 0.367 \\
\hline \hline
\end{tabular}

Table 1: The means and standard errors of all three static conditions for each of the dependent variables.

\begin{tabular}{|c|c|c|c|c|}
\hline $\begin{array}{c}\text { Dependent } \\
\text { Variable }\end{array}$ & Effects & DF & F Value & $\operatorname{Pr}>\mathbf{F}$ \\
\hline \multirow{4}{*}{ RMS Sway (cm) } & Condition & 2,57 & 10.43 & < <0.001* \\
\hline & 1 vs 2 & & & $0.001 *$ \\
\hline & 1 vs 3 & & & $<0.001^{*}$ \\
\hline & 2 vs 3 & & & 0.911 \\
\hline \multirow{4}{*}{$\begin{array}{c}\text { Strategy Score } \\
(\%)\end{array}$} & Condition & 2,57 & 4.67 & $0.013 *$ \\
\hline & 1 vs 2 & & & $0.018^{*}$ \\
\hline & 1 vs 3 & & & $0.048 *$ \\
\hline & 2 vs 3 & & & 0.915 \\
\hline \multirow{4}{*}{ MPF (Hz) } & Condition & 2,57 & 0.35 & 0.708 \\
\hline & 1 vs 2 & & & 0.964 \\
\hline & 1 vs 3 & & & 0.842 \\
\hline & 2 vs 3 & & & 0.695 \\
\hline \multirow{4}{*}{$\begin{array}{c}\text { Shift From Zero } \\
\text { (cm) }\end{array}$} & Condition & 2,57 & 0.54 & 0.583 \\
\hline & 1 vs 2 & & & 0.885 \\
\hline & 1 vs 3 & & & 0.554 \\
\hline & 2 vs 3 & & & 0.837 \\
\hline
\end{tabular}

Table 2: The main effects of condition as well as the pairwise comparisons of the static conditions ( $p r e N V=1, \operatorname{preVib}=2$, and postVib $=3$ ) for all dependent variables. * Denotes statistical significance.

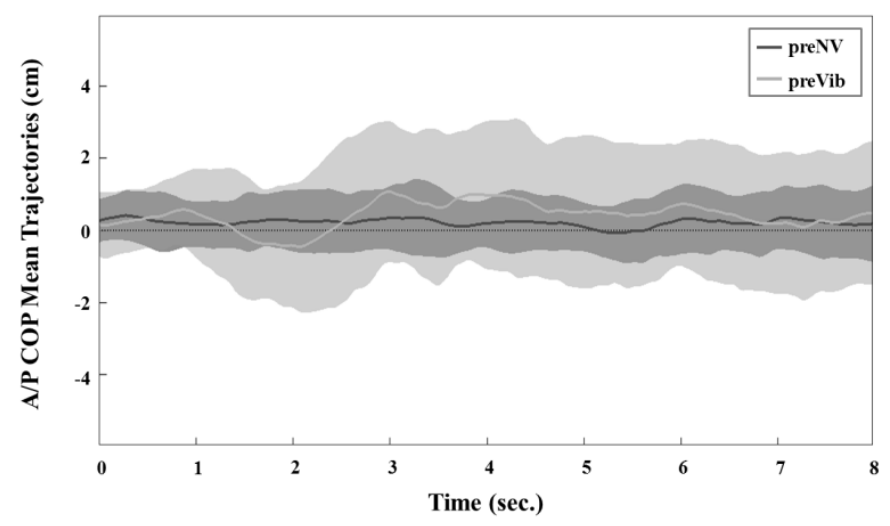

Figure 1: The A/P COP mean trajectories (solid lines) for all 20 subjects \pm 1 standard deviation (shaded areas) of the mean across the eight seconds of data analyzed in the pre no vibration (preNV) and pre vibration (preVib) conditions. Time zero indicates the time at which vibration was initiated. Positive trajectories represent anterior sway, while negative trajectories depict posterior sway. Note that vibration generated a greater amount of variability in the mean trajectories. 


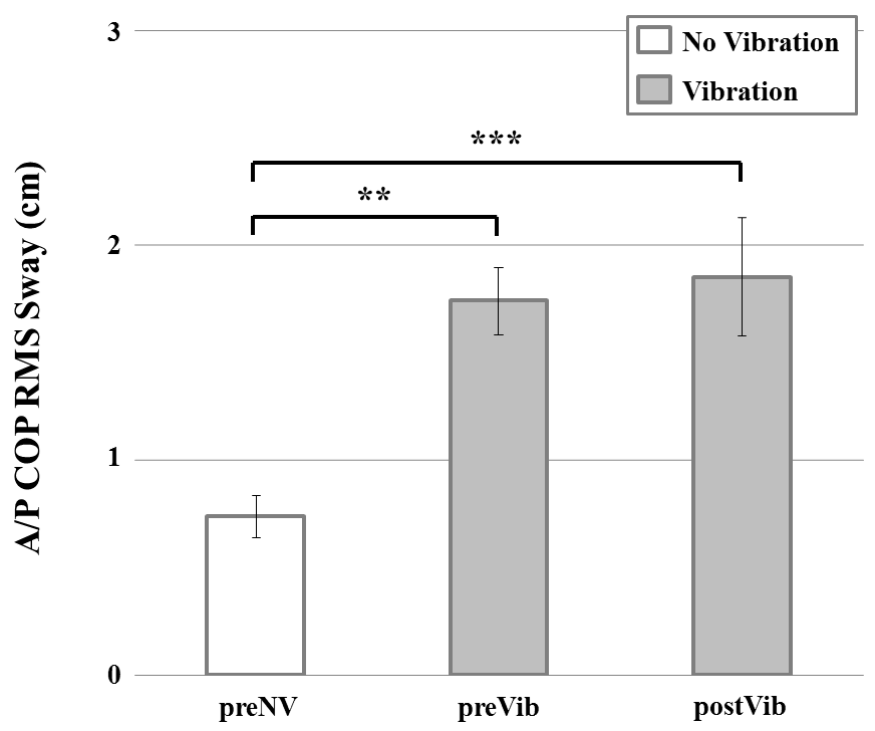

Figure 2: A bar graph showing means for the RMS of the A/P sway during the two pre-tests as well as the post-test. The mean for the pre-test trial without vibration (preNV) is represented by the white bar, while the shaded bars represent pre- and post-test means for the vibration trials (preVib and postVib). Asterisks denote significance leves: $* p<0.05$, ** $p<0.01$, and $* * * p<0.001$.

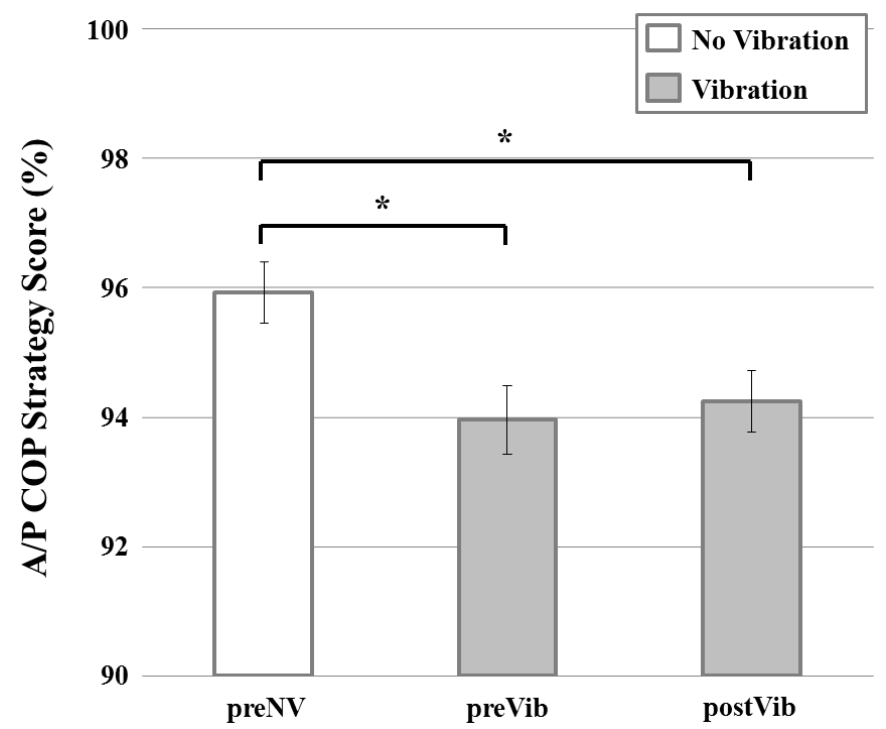

Figure 3: A bar graph showing means for the strategy score percentage during the two pre-tests as well as the post-test. The mean for the pre-test trial without vibration (preNV) is represented by the white bar, while the shaded bars represent pre- and post-test means for the vibration trials (preVib and postVib). Asterisks denote significance leves: $* p<0.05$, ** $p<0.01$, and $* * * p<0.001$

\section{Discussion}

The significant results from the RMS sway and strategy score measures depicted in figure 2 and figure 3 respectively are indicative of postural responses to TA vibration. Significantly greater RMS sway values for the TA vibration conditions (preVib and postVib) as compared to the pretest condition without vibration (preNV) represent a TA vibration response to quiet stance in which greater $\mathrm{A} / \mathrm{P} \mathrm{COP}$ sway magnitudes were recorded. The result indicating no significant changes in RMS sway for preVib and postVib conditions suggests that fatigue was not a major factor in the vibration responses, even if fatigue was not directly measured. The increased variability (i.e., sway) in A/P COP position can be further visualized by observing the mean $\mathrm{A} / \mathrm{P} \mathrm{COP}$ trajectories of the preNV and preVib conditions in figure 1. In addition, long latency (observed approximately $2 \mathrm{~s}$ after the onset of vibration in figure 1) of variability in vibration responses suggests that these responses are not compatible with reflex contribution in muscle spindle activation, which is normally observed in less than $100 \mathrm{~ms}$ [15]. Significantly lower strategy score percentages observed for the TA vibration conditions (preVib and postVib) as compared to the pretest condition with no vibration (preNV) also support findings of a TA vibration postural response. As stated earlier, lower strategy scores depict greater shear forces being generated and tend to represent increased motion about the hips relative to ankle strategies. However, due to the relatively high strategy scores $(>90 \%)$ for all static conditions and consistent with previous research on static stance [17], ankle strategies were still the dominant strategy utilized for $\mathrm{A} / \mathrm{P}$ postural control during quiet stance, despite the presence of vibration. Significantly lower strategy scores for the TA vibration conditions as compared to no vibration do however, reflect the fact that greater shear forces were required to maintain stable upright stance when vibration was applied to the TA.

As reported in previous research, the somatosensory input provided by Type Ia afferents affected by TA vibration likely contributes to the increased $\mathrm{A} / \mathrm{P}$ COP sway magnitudes observed in the vibration conditions [3]. Interestingly, although means of the A/P COP shift from zero positions seen in Table 1 were larger and thus more anterior for vibration conditions preVib (84\% more) and postVib (184\% more) than the condition without vibration (preNV), these differences did not reach statistical significance. This is likely due to the larger variability observed in measures associated with the two vibration conditions. Other possible explanations might include a small sample size, few repetitions performed, or potentially the short duration of TA vibration. In addition, shift from zero takes into account mean amplitude for the entire trajectories as opposed to the peak amplitude which has been evaluated in previous studies [17]. 
Non-significant results for MPF reveal that sway frequency oscillations did not significantly vary among the three conditions (preNV, preVib, and postVib) indicating that vibration did not influence the frequency oscillatory properties of the COP motion. Furthermore, repeated exposure to vibration did not impact MPF indicating that potentially disrupted sensory input did not exert influence on this postural control measure over time. This finding is in agreement with previous research that cutaneous vibration applied to the skin over the torso does not appear to induce a disruptive increase in sway frequency [5], implying that the frequency response to TA muscle vibration also remains within the range of normal sway. This may have clinical implications because short-term muscle vibration could be used to indicate the direction of body tilt to maintain a stable standing posture as cutaneous vibration has been used as "alarm" signals for balance related rehabilitation training [24, 25]. Furthermore, involuntary postural response to TA muscle vibration revealed in the present study may leverage voluntary action of prime mover muscles to facilitate postural regulation in individuals with neuromuscular disease.

Despite previous studies indicating that adaptation to the vibration response can occur $[3,17]$, our results seem to support our hypothesis that the vibration response during quiet stance remains relatively unchanged despite the repeated exposures to vibration during the perturbation trials. No statistically significant differences were found between the preand post-test means of the two static vibration conditions (preVib and postVib) for any of the dependent variables. The current results suggest that the disruptive sensory input that presumably was responsible for the observed differences between the no vibration and the vibration conditions was not fully ignored (i.e. down weighted) despite repeated exposure during experimental conditions involving upright stance. During this task, muscle spindles of TA muscles likely encoded spatial representation of the body sway as evidenced by the significant changes in COP motion, but the sway associated with the altered sensory feedback was not so disruptive (inducing loss of balance) as to require an extensive down weighting of the sensory input. In other words, although increased sway resulted from vibration, the extent of the increased sway did not threaten postural stability as would be reflected in a fall. Thus, the context associated with this particular postural control task dictated that there was no need to down weight the altered sensory input in order to complete the task.
Given there was no compelling reason to fully 'ignore' the altered sensory input during the first exposure to vibration, there would be no expectation that the post vibration condition would produce different results and that is what was observed in the current data (i.e. no significant differences between the preVib and postVib measures). Having seen no evidence of adaptation to repeated exposures to vibration in this study does not suggest that other tasks involving vibration would not produce a down weighting of altered sensory input depending upon the context. Should the effects of vibration place a person at risk for harm or impair his or her ability to effectively complete a given task, it is likely that adaptations to repeated vibration exposure would be observed. Changing the context such as imposing longer durations of vibration exposure would conceivably result in observable adaptations as well.

In terms of utilizing TA vibration for therapeutic or training purposes, this study suggests that repeated exposures delivered within the same context as given in this experiment do not seem to interfere with the maintenance of upright balance in quiet stance. It also suggests that the observed postural responses to TA vibration (such as a more anterior sway) during quiet stance may remain relatively similar under the same conditions as this study, although it was noted that variability in COP trajectories does increase with application of the vibration stimulus.

\section{ACKNOWLEDGEMENTS}

The authors would like to thank Sara Lalani, Brett Siders, and Recep Ozdemir for their contributions to the project.

\section{REFERENCES}

[1] J. Massion, "Postural changes accompanying voluntary movements. Normal and pathological aspects," Hum Neurobiol, vol. 2, no. 4, pp. 261-267, Jan. 1984.

[2] H. Slijper and M. L. Latash, "The effects of muscle vibration on anticipatory postural adjustments," Brain Res, vol. 1015, no. 1-2, pp. 57-72, Jul. 2004.

[3] M. Dettmer, A. Pourmoghaddam, D. P. O'Connor, and C. S. Layne, "Interaction of support surface stability and Achilles tendon vibration during a postural adaptation task," Hum Mov Sci, vol. 32, no. 1, pp. 214-227, Feb. 2013.

[4] F. B. Horak, "Postural orientation and equilibrium: What do we need to know about neural control of balance to prevent falls?," Age Ageing, vol. 35-S2, pp. ii7-ii11, Sep. 2006.

[5] B.C. Lee, B. J. Martin, and K. H. Sienko, "Directional postural responses induced by vibrotactile stimulations applied to the torso," Exp Brain Res, vol. 222, no. 4, pp. 471-482, Oct. 2012. 
[6] L. M. Nashner, "Fixed patterns of rapid postural responses among leg muscles during stance," Exp Brain Res, vol. 30, no. 1, pp. 13-24, Oct. 1977.

[7] A. Priplata, J. Niemi, M. Salen, J. Harry, L. A. Lipsitz, and J. J. Collins, "Noise-Enhanced Human Balance Control," Phys Rev Lett, vol. 89 , no. 23 , p. 238101 , Nov. 2002

[8] A. L. Smiley-Oyen, H.-Y. K. Cheng, L. D. Latt, and M. S. Redfern, "Adaptation of vibration-induced postural sway in individuals with Parkinson's disease," Gait Posture, vol. 16, no. 2, pp. 188-197, Oct. 2002.

[9] S. Carver, T. Kiemel, and J. J. Jeka, "Modeling the dynamics of sensory reweighting," Biol Cybern, vol. 95, no. 2, pp. 123-134, Aug. 2006 .

[10] H. Ceyte, C. Cian, R. Zory, P.-A. Barraud, A. Roux, and M. Guerraz, "Effect of Achilles tendon vibration on postural orientation," Neurosci Lett, vol. 416, no. 1, pp. 71-75, Apr. 2007.

[11] G. Eklund and K. E. Hagbarth, "Normal variability of tonic vibration reflexes in man," Exp Neurol, vol. 16, pp. 80-92, 1966.

[12] G. Eklund, "General features of vibration-induced effects on balance," Ups J Med Sci, vol. 77, pp. 112-124, 1972.

[13] T. Lapole and C. Pérot, "Effects of repeated Achilles tendon vibration on triceps surae force production," $J$ Electromyogr Kinesiol, vol. 20, no. 4, pp. 648-654, Aug. 2010.

[14] M. A. Lebedev and A. V Polyakov, "Analysis of surface EMG of human soleus muscle subjected to vibration," $J$ Electromyogr Kinesiol, vol. 2, no. 1, pp. 26-35, Jan. 1992.

[15] B. J. Martin and H. S. Park, "Analysis of the tonic vibration reflex: Influence of vibration variables on motor unit synchronization and fatigue," Eur J Appl Physiol Occup Physiol, vol. 75, no. 6, pp. 504511, Jan. 1997.

[16] H. S. Park and B. J. Martin, "Contribution of the tonic vibration reflex to muscle stress and muscle fatigue," Scand J Work Environ Health, vol. 19, no. 1, pp. 35-42, Mar. 1993.

[17] S. Caudron, L. Langlois, V. Nougier, and M. Guerraz, "Attenuation of the evoked responses with repeated exposure to proprioceptive disturbances is muscle specific," Gait Posture, vol. 32, no. 2, pp. 161-168, Jun. 2010.

[18] V. Hatzitaki, M. Pavlou, and A. M. Bronstein, "The integration of multiple proprioceptive information: Effect of ankle tendon vibration on postural responses to platform tilt," Exp Brain Res, vol. 154, no. 3, pp. 345-354, Mar. 2004.

[19] C. Thompson, M. Bélanger, and J. Fung, "Effects of bilateral Achilles tendon vibration on postural orientation and balance during standing," Clin Neurophysiol, vol. 118, no. 11, pp. 2456-2467, Nov. 2007.

[20] Y. P. Ivanenko, V. L. Talis, and O. V Kazennikov, "Support stability influences postural responses to muscle vibration in humans," Eur $J$ Neurosci, vol. 11, no. 2, pp. 647-654, Mar. 1999.

[21] N. Vuillerme, F. Danion, N. Forestier, and V. Nougier, "Postural sway under muscle vibration and muscle fatigue in humans," Neurosci Lett, vol. 333, no. 2, pp. 131-135, Nov. 2002.
[22] NeuroCom. "Balance manager systems instructions for use," Clackamas, OR: NeuroCom International, 2008.

[23] NeuroCom. "Balance manager systems clinical interpretation guide," Clackamas, OR: NeuroCom International, 2009.

[24] B. C. Lee, J. Kim, S. Chen, and K. H. Sienko, "Cell phone based balance transfer," J Neuroeng Rehabil, vol. 9, no. 10, Feb. 2012.

[25] K. H. Sienko, M. D. Balkwill, L. I. Oddsson, and C. Wall, "Effects of multi-directional vibrotactile feedback on vestibular-deficient postural performance during continuous multi-directional support surface perturbations," J Vestib Res, vol. 18, no. 5-6, pp. 273-285, Jan 2008. 